\title{
«Cachez cette erreur que je ne saurais voir ! »
}

Représentations et attitudes normatives chez des enseignants et apprenants de français langue étrangère

Hide this Error that I dare not see! Representations of Deviant Forms and Manifestations of Linguistic and Communicative Consciousness among Teachers and Students of French as a Foreign Language

Jean-Marc Defays et Deborah Meunier

\section{(2) OpenEdition}

Journals

\section{Édition électronique}

URL : http://journals.openedition.org/pratiques/2752

DOI : $10.4000 /$ pratiques. 2752

ISSN : 2425-2042

\section{Éditeur}

Centre de recherche sur les médiations (CREM)

\section{Référence électronique}

Jean-Marc Defays et Deborah Meunier, « «Cachez cette erreur que je ne saurais voir ! » », Pratiques [En ligne], 167-168 | 2015, mis en ligne le 01 avril 2016, consulté le 14 novembre 2019. URL : http:// journals.openedition.org/pratiques/2752; DOI : 10.4000/pratiques.2752

Ce document a été généré automatiquement le 14 novembre 2019.

(C) Tous droits réservés 


\section{«Cachez cette erreur que je ne saurais voir!»}

Représentations et attitudes normatives chez des enseignants et apprenants de français langue étrangère

Hide this Error that I dare not see! Representations of Deviant Forms and Manifestations of Linguistic and Communicative Consciousness among Teachers and Students of French as a Foreign Language

Jean-Marc Defays et Deborah Meunier

\section{Introduction}

1 On sait que la question du traitement à réserver aux «erreurs" a donné lieu à des réponses très différentes au cours de l'histoire de la didactique des langues étrangères. Après avoir rapidement résumé ces différentes options, nous nous intéresserons au traitement, dans le cadre d'un enseignement des langues étrangères de type communicatif, des erreurs et des déviances définies par un locuteur en fonction de la norme scolaire enseignée et/ou d'un français de référence (Morin, 2000) imaginé. Nous postulons que c'est lors des interactions en classe que les discriminations - au niveau des représentations comme des pratiques, des enseignants comme des apprenants - se créent, se confirment, se corrigent, se nuancent entre les formes acceptables ou non.

2 Dès lors, nous nous proposons ici, en un premier stade, de recueillir, d'analyser, de comparer les représentations d'enseignants de français langue étrangère et de leurs apprenants, pour la plupart des étudiants Erasmus, que nous avons interrogés sur leurs représentations des erreurs/déviances commises ou rencontrées (formes et pratiques incorrectes, déviantes, variantes, approximatives, provisoires...) et de la/des norme(s) qui leur ser(ven)t de référence pour en juger.

3 À un second stade, nous étudierons leurs attitudes et nous verrons à quels niveaux se manifeste la conscience linguistique de ces enseignants et apprenants en les exposant à 
une série d'énoncés considérés communément comme des " déviances-types $»^{1}$ (d'ordre phonétique, lexical, syntaxique, orthographique, discursif, pragmatique...), qu'ils devront repérer, identifier, évaluer. Par la même occasion, nous interrogerons la prégnance du clivage langue/communication chez nos témoins.

\section{L'erreur en didactique des langues}

\subsection{De la « faute » à « l'erreur »}

4 Selon les conceptions qui sous-tendaient la traditionnelle méthode grammairetraduction, les erreurs étaient des fautes qu'il fallait sanctionner (et donc exclure) pour qu'elles ne se produisent plus, tandis que l'approche structurobéhavioriste, qui s'est présentée comme scientifique, tentait de les prévenir à l'aide d'exercices spécifiques qui devaient neutraliser les interférences de la langue-source vers la langue-cible. On considérait en effet que la plupart des erreurs provenaient de ces transferts qui nuisaient à l'apprentissage et qu'il fallait à tout prix éviter.

5 Plus tard, l'approche communicative a adopté une conception de l'erreur radicalement différente: la nécessité de la communication étant plus importante que le souci de correction de la langue, le professeur n'intervient à propos de celle-ci que si elle contrarie celle-là : il n'y a donc plus de "fautes », mais seulement des « erreurs » qui sont toujours relatives. D'autant plus relatives que la théorie des stades d'acquisition linguistique ( Processability theory, Pienemann, 1998) a montré qu'une erreur de compétence (à différencier de l'erreur de performance due à des facteurs non linguistiques) est logique par rapport au système linguistique transitoire, l'interlangue, dans lequel évolue à ce moment de son apprentissage l'apprenant qui la commet. Une erreur est ainsi non seulement inévitable, mais normale, prévisible, et surtout incontrôlable et incorrigible isolément et immédiatement puisqu'elle relève de tout le (micro)système de l'interlangue.

6 Dans une perspective cognitive, l'erreur constitue d'abord une preuve que l'apprentissage est en progrès, puisque l'acquisition d'une langue suppose que l'apprenant élabore et vérifie des hypothèses sur son fonctionnement; on ne peut pas apprendre une langue si on ne prend pas le risque de se tromper. Ces erreurs constituent la source et le matériau de l'apprentissage (approche « intégrative ») en fonction de la réaction de l'interlocuteur au cours de la communication, mais également en classe, quand l'enseignant profite des difficultés de ses apprenants pour développer la réflexion métalinguistique.

On ne parle donc plus de «faute» en didactique des langues, car ce terme envisage la question de manière binaire, opposant absolument et définitivement une prestation (orale ou écrite, lors de la compréhension comme de la production) soi-disant « incorrecte » à une prestation soi-disant " correcte ", alors que l'« erreur " est toujours corrélative de la grammaire, du dictionnaire, du registre de langue, du contexte et des intentions de la communication, du niveau de l'apprenant... envisagés. Variante ou déviance, l'erreur fait en tout cas partie intégrante de l'apprentissage et de l'enseignement d'une langue étrangère. C'est en tout cas ce qu'en dit actuellement la didactique ; nous allons voir si concordent les représentations qu'en ont les apprenants et leurs enseignants. 


\subsection{Typologie et traitement des erreurs}

8 On a établi plusieurs typologies, par exemple selon l'aspect de la langue - phonologique, morphosyntaxique, lexical, socioculturel, etc.- ou selon l'habileté communicative - écouter, parler, lire, écrire - qu'elles affectent. On distingue aussi les erreurs dues à une omission ( $*$ *je ne le crois »), à un ajout ( * *je n'ai pas vu personne »), à une substitution ( * je non le crois pas») ou à un déplacement («*je ne l'ai cru pas»). On différencie encore divers degrés de gravité des erreurs : certaines s'assimilent à des approximations peu compromettantes, tandis que d'autres créent des contresens complets. Des erreurs ont un caractère systématique et relèvent de la compétence de l'apprenant (c'est le cas des erreurs fossilisées); d'autres sont occasionnelles et seulement liées aux conditions de sa performance (inattention, fatigue, émotion). Enfin, on classe les erreurs selon leurs causes : certaines proviennent de l'influence de la langue-source (interlinguales), d'autres d'une mauvaise maitrise de la langue-cible (intralinguales: simplification ou surgénéralisation d'une règle, par exemple). L'enseignant tient compte de ces typologies quand il se propose de corriger une erreur : selon les cas, il interviendra au moment où elle est commise ou plus tard, en la signalant seulement ou en la corrigeant aussi, en la corrigeant lui-même ou en amenant l'apprenant à se corriger, en se limitant à la forme concernée ou en la replaçant dans son système (grammatical, lexical, discursif).

D'une manière générale, on préconise aujourd'hui d'adapter d'abord le signalement et la correction de ces erreurs au niveau de la maitrise de la langue et au degré de sécurité des apprenants. Un débutant, plus crispé, acceptera sans doute moins bien une correction systématique qu'un apprenant d'un niveau plus élevé qui, au contraire, l'attend de son professeur. D'autre part, la réaction à l'erreur devrait être proportionnelle non pas à sa gravité dans l'absolu, mais à sa portée dans la communication en cours que la correction devrait faciliter et non gêner. Pendant une activité d'expression orale, par exemple, l'enseignant n'interviendra que pour des erreurs qui constituent un obstacle à l'intercompréhension, et il évitera les longues explications qui risquent de décourager les apprenants et qu'il réservera pour une séance spéciale. Par ailleurs, on privilégiera la correction des erreurs systématiques, sur lesquelles les explications et les exercices ont prise, sans trop se soucier des erreurs occasionnelles dues à la distraction ou au stress. Enfin et surtout, on déculpabilisera les étudiants en insistant sur l'intérêt que représentent leurs erreurs, et en les invitant à les analyser eux-mêmes avant de les corriger, et ensuite à trouver des moyens (métalinguistiques, métacognitifs) pour qu'elles se reproduisent de moins en moins.

\section{Représentations linguistiques, attitudes et pratique corrective}

10 Nous utilisons la notion de représentation dans le contexte restreint de l'enseignement/ apprentissage du français langue étrangère (FLE), c'est-à-dire que nous nous intéressons aux représentations sur les langues (et ceux qui les parlent), ce que nous appelons des représentations linguistiques, dont la nature est à la fois cognitive, discursive et sociale et qui s'activent (déjà-là) ou s'élaborent discursivement (de façon explicite ou implicite) à partir de praxis sociales et scolaires, de même qu'elles permettent à l'individu de construire et d'organiser sa connaissance de la réalité linguistique (Meunier, 2012). 
Les représentations, via le métalangage dans sa fonction d'opérateur de représentations, structurent le processus d'acquisition langagière et culturelle (Boyer \& Peytard, 1990 ; Dabène, 1997), constituent un "horizon d'attente» déterminant pour les acteurs didactiques et agissent sur les comportements discursifs, cognitifs (Beacco, 2004) et interculturels (Dervin, 2008).

Dans le cas qui nous occupe, c'est précisément la dimension normative des représentations qui nous intéresse, dans la mesure où le discours normatif ${ }^{2}$, dans sa dimension processuelle (la norme est processus d'évaluation d'après B. Py, 2000) comme dans l'énonciation du résultat de l'inventaire de ce qui est correct ou ne l'est pas (le produit selon B. Py), nous informe sur les attitudes adoptées par les locuteurs vis-à-vis des pratiques, acceptables ou en marge, celles qu'on se doit de reproduire, d'apprendre, d'enseigner, ou au contraire d'" exclure».

La conscience normative des apprenants d'une langue étrangère, qu'elle soit observable lors d'interactions où elle se réalise comme processus en temps réel, ou via les discours tenus sur la norme (temps différé), comme c'est le cas dans l'étude présentée ici, peut être sollicitée afin d'éclairer certaines attitudes évaluatives ${ }^{3}$ des apprenants de langue.

En effet, tout locuteur, même débutant dans une langue étrangère, développe une pratique corrective qui sera conditionnée par ses représentations. Pierre Bourdieu (2000) parlait de l'«inconscient d'école», cet arbitraire incorporé par les membres du système scolaire, une doxa commune en œuvre dans le geste correctif. Cette pratique varie selon l'ensemble des compétences linguistiques de l'individu et selon sa culture discursive, une sorte de filtre qui définit ce qu'on (ne) doit (pas) dire et de quelle manière. Enfin, la pratique corrective se manifeste par des comportements évaluatifs que le locuteur développe vis-à-vis de ses propres productions (autocorrection) ou de celles des autres (hétérocorrection). Cette pratique corrective pourra se penser sous forme de continuum:

Figure 1. Continuum de la pratique corrective (Meunier, 2013)

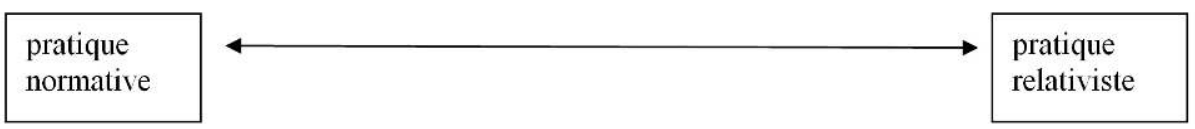

14 Les représentations linguistiques, les pratiques déclarées, vont donc organiser la perception des réalités et vont guider les conduites, les pratiques effectives, notamment d'évaluation, lesquelles s'expriment à travers les discours tenus sur la langue, mais aussi les attitudes adoptées par rapport à des énoncés dans le cas par exemple des jugements d'acceptabilité.

\section{Méthode, objectifs et limites de l'enquête}

Les résultats présentés ici sont issus d'une enquête exploratoire menée à l'Institut supérieur des langues vivantes de l'Université de Liège en mai 2013 auprès de 11 enseignants de FLE s'adressant à des publics d'étudiants Erasmus de niveaux différents, et qui ont un nombre d'années d'expérience variable dans l'enseignement du FLE. Par ailleurs, nous avons interrogé 57 apprenants de niveaux différents (B1+, B2, B2+, C1). Nous avons fait circuler deux questionnaires (en annexe) identiques parmi les enseignants et leurs apprenants. 

enquête qualitative préliminaire qui a permis de déterminer les observables à partir du terrain, des dires des acteurs. Notons que cette démarche s'inscrit dans une perspective sociodidactique (au sens que lui donne P. Blanchet, 2011) et a permis d'éviter de tomber dans l'écueil classique des enquêtes quantitatives dont les questions fermées induisent les réponses obtenues et reflètent davantage les hypothèses à priori du chercheur plutôt que les représentations des enquêtés.

Le premier questionnaire consistait en une liste d'énoncés « déclencheurs " par rapport auxquels les participants devaient exprimer leur accord ou leur désaccord, de façon catégorique ou plutôt mesurée. Cette première étape de l'enquête a permis de voir s'ils adoptaient une posture plutôt normative et pre-/proscriptive, ou au contraire tolérante et relativiste vis-à-vis des «normes » qui leur étaient proposées. Ces affirmations portaient sur plusieurs aspects $d u$ «bon usage » imaginé de la langue: les critères strictement linguistiques; les aspects fonctionnels et sociaux de la langue; les critères spécifiques aux usages des francophones de Belgique.

Le second questionnaire consistait en une liste de 30 énoncés potentiellement « déviants» ou « incorrects » sur les plans morphosyntaxique, orthographique, lexical, pragmatique et sociolinguistique. Les participants devaient juger du degré d'acceptabilité des énoncés. Cette deuxième étape a permis de mettre en évidence la sensibilité des sujets par rapport aux différents types de "déviances » et de déterminer si leur attitude était plus ou moins normative, comparativement à leurs représentations.

19 Nous avons donc pu comparer les postures dominantes chez les enseignants et les apprenants, tant au niveau de leurs représentations à priori que de leurs attitudes effectives d'évaluation.

20 Cependant, cette enquête présente plusieurs limites. D'abord, le nombre de professeurs interrogés étant limité, il est difficile de prétendre à la représentativité de nos observations. Mais une enquête de plus grande ampleur permettrait facilement de confirmer, infirmer, ou nuancer nos conclusions.

21 Par ailleurs, les données obtenues par questionnaires devraient par la suite être complétées par une étude qualitative, sous forme d'entretiens individuels ou de discussions de groupe, qui permettrait de faire émerger les représentations discursives au fil de l'entretien de façon plus spontanée (même si le guide d'entretien porte lui aussi la trace de la subjectivité de son concepteur et que le contexte de l'entretien est un biais en soi...). Ce complément d'enquête reste à réaliser et devrait nous éclairer davantage sur les premiers résultats obtenus à ce stade de la recherche.

22 Enfin, nous n'avons pas eu l'occasion d'explorer ici toutes les variables susceptibles d'intervenir au niveau des représentations et des attitudes. En effet, il serait intéressant de voir sur un échantillon plus important de professeurs si les différences de niveaux du public d'apprenants, ou encore le nombre d'années d'expérience dans l'enseignement du FLE influencent leurs représentations et attitudes face aux erreurs. De même pour les étudiants: quel est l'impact du niveau des apprenants sur leurs représentations et attitudes? 


\section{Les résultats}

\subsection{Représentations et attitudes face aux formes/pratiques « à exclure»}

Les enseignants et les apprenants se sont montrés en général plus normatifs que tolérants au niveau de leurs représentations. On observe une faible différence entre les deux groupes : les enseignants sont légèrement moins normatifs (54\%) que les étudiants (68 \% ). On peut voir dans ces chiffres le reflet du souci accru de la norme dû à un sentiment d'incompétence et d'insécurité chez les étudiants. Ce constat est confirmé par les travaux de V. Castellotti (2012), D. Meunier (2013) ou D. Meunier et L. Rosier (2014) sur la figure du non-natif dans l'imaginaire linguistique et le sentiment de non-légitimité qui accentue le besoin de recourir au discours normatif, par exemple via les outils du savoir comme les grammaires, les dictionnaires ou le Bescherelle.

$\mathrm{Au}$ niveau des attitudes, on remarque une inversion des tendances observées précédemment : ce sont les enseignants qui sont plus normatifs (56\%) que les étudiants (42\%). Chez les enseignants, les attitudes et les représentations correspondent : ils sont plus normatifs que relativistes; chez les étudiants, les attitudes et les représentations divergent : ils sont plus normatifs dans leurs représentations que dans leurs attitudes.

Figure 2. Comparaison des attitudes et des représentations chez les enseignants

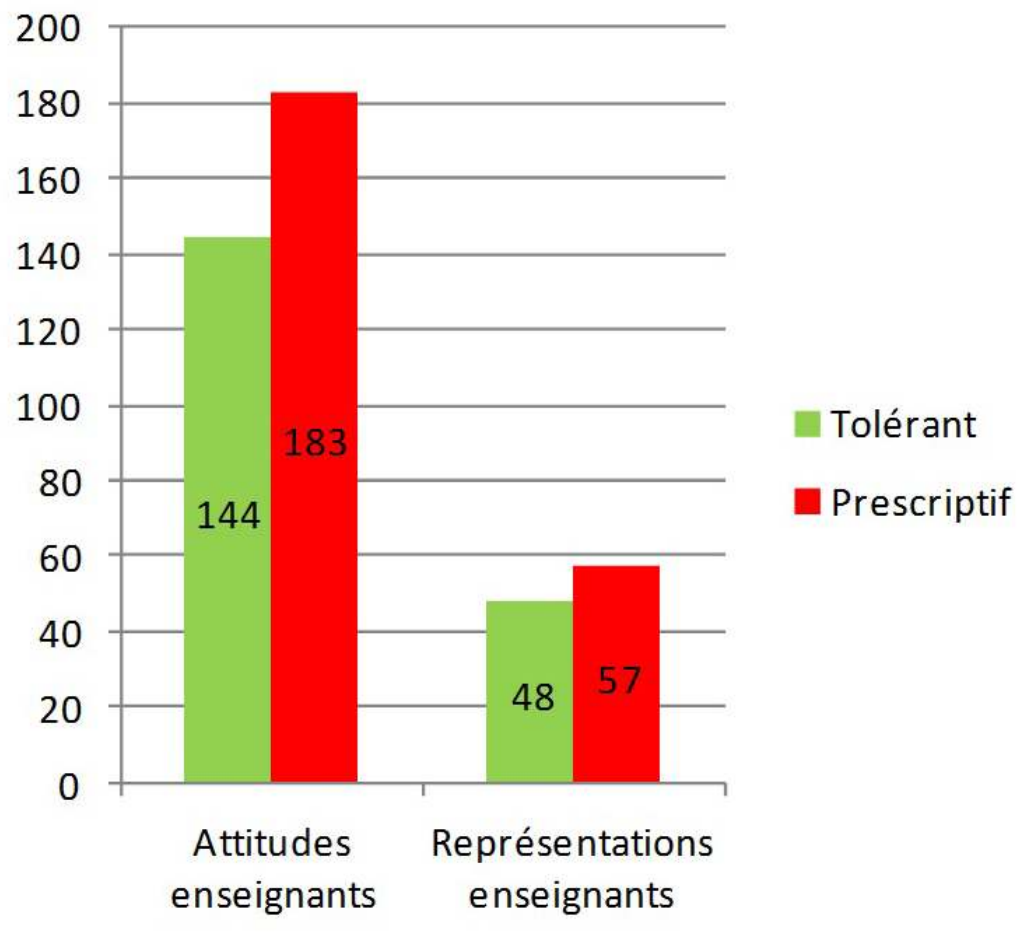


Figure 3. Comparaison des attitudes et des représentations chez les étudiants

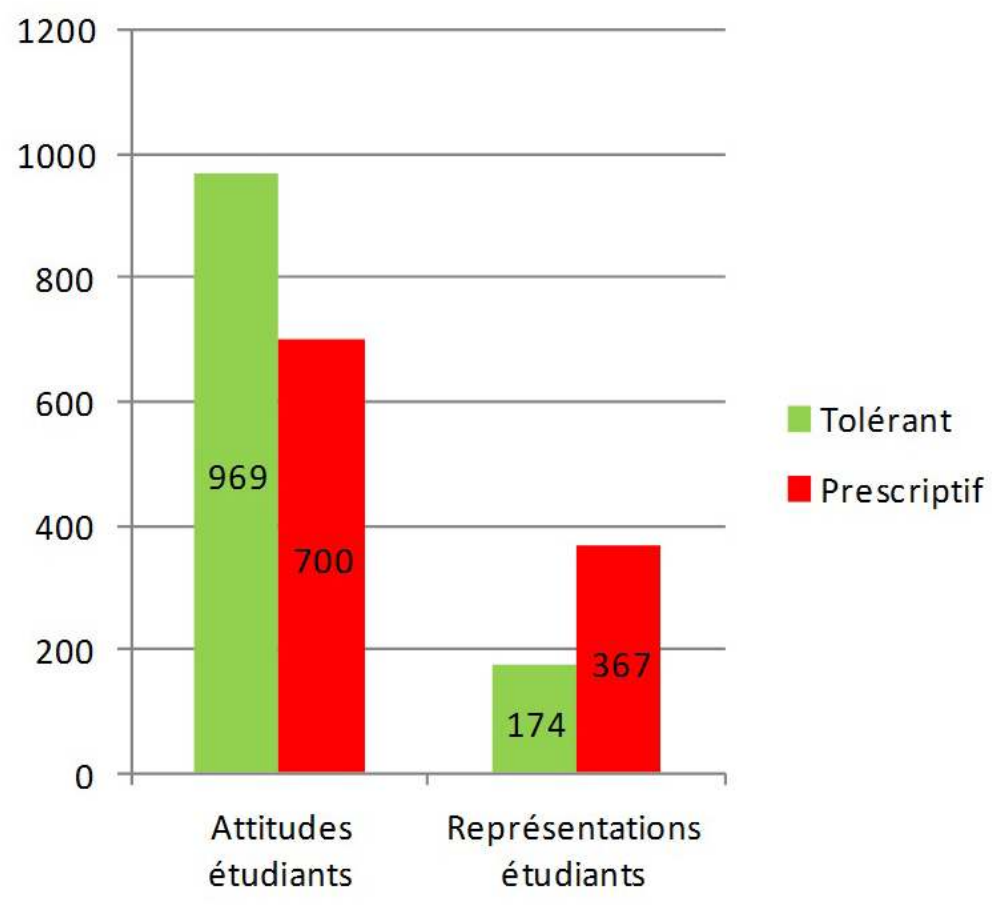

25 Selon nous, l'attitude plus tolérante/relativiste des étudiants peut s'expliquer par leur niveau de compétence linguistique, selon toute logique moins élevé que celui des enseignants. Les deux groupes ne jouant pas «à armes égales », il est donc évidemment difficile de conclure de cette différence chiffrée une différence d'ordre représentationnel qui conditionnerait l'attitude observée.

Par contre, si l'on analyse les différents types de "déviances " et les représentations/ attitudes par rapport aux différents aspects de l'usage de la langue, on constate des différences intéressantes au sein de chaque groupe et entre les deux groupes.

\subsection{Comparaison des représentations chez les enseignants et les étudiants concernant les différents aspects du « bon usage » du français}

C'est au niveau de la syntaxe et de l'orthographe, lieux de cristallisation du discours normatif par excellence, et des aspects communicatifs que les deux groupes sont les plus normatifs.

Tableau 1. Déclinaison de la posture normative chez les enseignants et les étudiants

\begin{tabular}{|l|l|l|}
\hline & \multicolumn{2}{|l|}{ Posture normative } \\
\hline \multirow{3}{*}{ Syntaxe } & $\begin{array}{l}\text { Enseignants } \\
100 \%\end{array}$ & $\begin{array}{l}\text { Étudiants } \\
98 \%\end{array}$ \\
\hline
\end{tabular}




\begin{tabular}{|l|l|l|}
\hline Orthographe & $\begin{array}{l}\text { Enseignants } \\
90 \%\end{array}$ & $\begin{array}{l}\text { Étudiants } \\
98 \%\end{array}$ \\
\hline Communication & $\begin{array}{l}\text { Enseignants } \\
100 \%\end{array}$ & $\begin{array}{l}\text { Étudiants } \\
96 \%\end{array}$ \\
\hline
\end{tabular}

Les étudiants vont être plus normatifs que les enseignants au niveau du lexique :

Figure 4. Normes lexicales

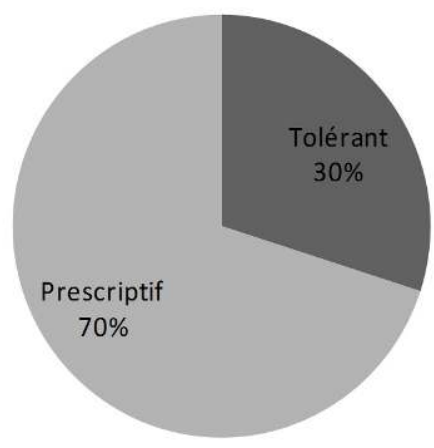

Normes lexicales. Enseignants

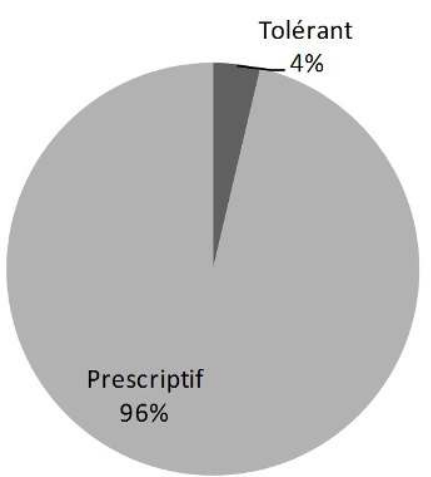

Normes lexicales. Étudiants

On observe la même tendance par rapport aux emprunts : dans $9 \%$ des cas, la pratique est prescriptive chez les enseignants, alors qu'elle l'est dans $54 \%$ des cas chez les étudiants. De même pour les belgicismes qui sont tolérés à $100 \%$ par les enseignants, et à $78 \%$ par les étudiants.

Par contre, les étudiants sont beaucoup plus tolérants que les enseignants quand il s'agit de l'accent étranger: les enseignants condamnent l'accent étranger dans $90 \%$ des cas alors que les étudiants ne le stigmatisent que dans $40 \%$ des cas.

De la même façon, les enseignants seront plus normatifs en ce qui concerne les aspects pragmatiques (56\%) que les étudiants (38\%).

\subsection{Comparaison des attitudes chez les enseignants et chez les étudiants concernant les « déviances-types »}

De manière générale, les enseignants sont plus normatifs que les étudiants, nous l'avons dit. Mais on observe des différences significatives au sein de chaque groupe : on notera que les étudiants sont plus normatifs concernant la syntaxe (61\%) (parce qu'ils repèrent plus aisément les anomalies?) et qu'ils sont plus tolérants concernant les variétés sociolinguistiques ( $70 \%$ - parce qu'ils ne sont pas toujours en mesure de repérer les variétés ?). Tandis que les enseignants sont légèrement plus normatifs concernant les déviances pragmatiques ( $73 \%)$; et beaucoup plus tolérants concernant la variation $(87 \%$ ). 
Enfin, on constate que les attitudes sont plus normatives que les représentations aux niveaux pragmatique et sociolinguistique. La tolérance déclarée n'est donc pas effective dans les pratiques d'évaluation.

\section{Conclusions}

Malgré son caractère exploratoire, notre enquête a permis de dégager plusieurs constats intéressants sur les représentations et attitudes plus ou moins normatives d'enseignants et d'apprenants de FLE :

1. les représentations sont plus normatives que les attitudes, sauf au niveau pragmatique et sociolinguistique. Ce qui signifie que les répondants se déclarent moins normatifs qu'ils ne le sont en réalité pour ces deux aspects liés à l'usage de la langue ;

2. les représentations et attitudes sont plus normatives au niveau syntaxique et morphologique, lieux de cristallisation du discours normatif scolaire par excellence;

3. l'attitude tolérante des étudiants semble liée à leur niveau de compétence linguistique qui détermine la pratique corrective ;

4. les étudiants se déclarent très normatifs par rapport aux emprunts, alors que dans la pratique, il est courant qu'ils alternent les codes linguistiques. En effet, le code-switching et le one-word-switch sont des pratiques avérées entre les étudiants Erasmus qui sont amenés à pratiquer une ou plusieurs langues véhiculaires pour communiquer (sur la communication interalloglotte, Behrent, 2007 ; sur la pratique plurilingue des étudiants Erasmus, Anquetil, 2011 ; Meunier, 2013) ;

5. enfin, les représentations des étudiants semblent plus relativistes concernant l'accent étranger, alors que dans une autre enquête (par entretiens), on a constaté que l'accent étranger était fortement stigmatisé et pouvait même influencer négativement la perception de la compétence globale d'un locuteur (Meunier, 2012, 2013). À ce niveau aussi, les représentations déclarées sont plus relativistes que les attitudes évaluatives.

\section{BIBLIOGRAPHIE}

ANQUETIL, M. (2011). « Erasmus, lieux d'écriture et écriture des lieux pour public nomade ». Études de linguistique appliquée 162, avril-juin, p. 191-206.

BEACCO, J.-C. (dir.) (2004). « Représentations métalinguistiques ordinaires et discours ». Langages 154.

BEHRENT, S. (2007). La Communication interalloglotte. Communiquer dans la langue cible commune. Paris : L'Harmattan.

BLANCHET, P. \& CHARDENET, P. (dirs) (2011). Guide pour la recherche en didactique des langues et des cultures. Approches contextualisées. Paris : Éditions des Archives contemporaines.

BouRdieu, P. (2000). «L'inconscient d'école ». Actes de la recherche en sciences sociales 135, p. 3-5.

Boyer, H., \& PEYTARD, J. (dirs) (1990). « Les représentations de la langue : approches sociolinguistiques ». Langue française 1, vol. 85. 
CASTELLOTti, V. (2011). « Natif, non natif ou plurilingue : dénativiser l'enseignement des langues? ». In: Dervin, F. \& Badrinathan, V. (dirs), L'Enseignant non natif : identités et légitimité dans l'enseignement-apprentissage des langues étrangères. Fernelmont : EME, p. 29-50.

DABÈNE, L. (1994). Repères sociolinguistiques pour l'enseignement des langues. Paris : Hachette.

DEFAYS, J.-M. (2003). Le Français langue étrangère et seconde. Enseignement et apprentissage. Sprimont : Pierre Mardaga.

DEFAYS, J.-M., SAENEN, F. (2011). « De l'incidence du contexte sur les choix orthographiques en FLE : étude de cas ». Travaux Neuchâtelois de Linguistique (TRANEL) 54, p. 129-146.

DERVIN, F. (2008). Métamorphoses identitaires en situation de mobilité. Turku : Presses universitaires de Turku. En ligne : http://www.doria.fi/bitstream/handle/10024/36411/B307.pdf?sequence=1 (consulté le 29/07/13).

MEUNIER, D. (2012). «Erasmus : une culture discursive mobile ». Le Discours et la langue 3.2, p. 137-156.

- (2013), Les Représentations linguistiques des étudiants Erasmus et la vision plurilingue européenne : normes, discours, apprentissages. Thèse de doctorat en langues et lettres. Université de Liège/ Université libre de Bruxelles.

MEUNIER, D. \& ROSIER, L. (2014). « Quand le savoir s'emmêle... La construction discursive de la norme chez les locuteurs non experts ». Les Carnets du Cediscor 12, p. 99-113.

MOLINER, P. (1996). Images et représentations sociales. Grenoble, Presses universitaires de Grenoble.

MORIN, Y.-C. (2000). «Le français de référence et les normes de prononciation ». Cahiers de l'Institut de linguistique de Louvain 1, vol. 26, p. 91-135.

PIENEMANN, M. (1998). Language Processing and Second Language Development : Processability Theory. Amsterdam : John Benjamins.

PY, B. (2000). « La construction interactive de la norme comme pratique et comme

représentation ». AILE 12. En ligne : http://aile.revues.org/1464 (consulté le 13/06/12).

\section{ANNEXES}

\section{Annexe 1. Questionnaire sur les représentations}

Bien utiliser la langue française, c'est :

1. Respecter l'orthographe

2. Utiliser un langage clair et précis

3. Avoir un vocabulaire riche et varié

4. Ne pas employer d'expressions typiquement belges (belgicismes)

5. N’utiliser que les mots du dictionnaire

6. Passer inaperçu dans tous les milieux

7. Appliquer les règles de grammaire

8. Parler sans accent

9. Pouvoir se faire comprendre de n'importe qui

10. Ne pas emprunter de mots à d'autres langues 
« Tout à fait d'accord »/ « D'accord »/ «Plus ou moins d'accord »/ « Pas du tout d'accord »/ «Je ne sais pas»

\section{Annexe 2: Questionnaire sur les attitudes}

Dites si vous acceptez ou non ces (30) énoncés (ordre aléatoire) :

\section{a) Syntaxe}

Ex :

Je n'ai pas d'une automobile.

C'est moi que je l'ai trouvé.

\section{b) Morphologie}

Ex :

Si Pierre a besoin de mon stylo, je lui le donne.

Pierre et toi, que disez-vous?

\section{c) Orthographe (usage, grammatical)}

Ex :

Pierre a donné une pomme a Marie.

Il y a cinqs étudiants dans la classe.

\section{d) Lexique}

Ex :

J'ai obtenu un cadeau pour mon anniversaire.

J'ai checké mes bagages avant de partir.

\section{e) Pragmatique}

Ex :

Allô! Bonjour Monsieur ! Comment tu t'appelles?

Merci pour votre invitation; on bouffe quand?

\section{f) Sociolinguistique (belgicismes)}

Ex :

Je n'ai pas le temps; je ne sais pas venir chez toi. Je vais au cinéma ; tu viens avec?

«J'accepte tout à fait "

"J'accepte avec certaines réserves "

«Je n'accepte pas" 


\section{NOTES}

1. Les énoncés ont été sélectionnés sur la base d'une enquête sociolinguistique que nous avons menée auparavant auprès d'enseignants et d'étudiants Erasmus en français langue étrangère, sur les difficultés de la langue française et les lieux de cristallisation de différents types d'erreurs (l'emploi du subjonctif, la prononciation, la variation diatopique francophone...).

2. Nous parlons du « discours normatif » tel que l'envisage D. Meunier dans sa thèse de doctorat (2013), à savoir une manifestation du discours métalinguistique, susceptible de véhiculer des savoirs savants mais aussi ordinaires, fondée sur des normes objectives et subjectives et qui revêt une double dimension prescriptive et proscriptive.

3. Nous entendons attitude au sens de comportement, de pratique, à savoir la dimension évaluative des représentations sociales selon P. Moliner (1996).

\section{RÉSUMÉS}

La question du traitement à réserver aux " erreurs » a donné lieu à des réponses très différentes au cours de l'histoire de la didactique des langues étrangères. Pour la méthode grammairetraduction, les erreurs étaient des fautes qu'il fallait sanctionner (et donc exclure) pour qu'elles ne se produisent plus, tandis que l'approche structuro-behavioriste tentait de les prévenir à l'aide d'exercices qui devaient neutraliser les interférences de la langue-source vers la languecible. Aujourd'hui, l'erreur constitue une preuve que l'apprentissage est en progrès, puisque l'acquisition d'une langue suppose que l'apprenant élabore et vérifie des hypothèses sur son fonctionnement. C'est précisément au traitement des erreurs dans une approche communicative que nous avons choisi de nous intéresser ici. Nous nous proposons - en un premier stade - d'étudier les représentations d'enseignants de FLES et de leurs apprenants, interrogés sur leur conception et leur attitude concernant des erreurs commises ou rencontrées, et la/des norme(s) qui leur ser(ven)t de référence. À un second stade, nous verrons à quels niveaux se manifeste leur "conscience linguistique» en les exposant à une série d'énoncés considérés communément comme erronés, des « déviances-types ", qu'ils devront repérer, identifier, évaluer. Par la même occasion, nous interrogerons la prégnance du clivage langue/communication chez nos témoins.

The question of how to handle "mistakes" or errors has given rise to very different answers over the course of the history of foreign language teaching. According to the grammar/translation method, mistakes or errors were faults that had to be counted wrong (and thus excluded from discourse) so they would not be repeated. The structural/behavioristic approach tried to prevent mistakes by means of exercises intended to neutralize interferences occurring between the source language and the target language. Today, error is the proof that learning is taking place. The acquisition of a foreign language presupposes that the learner form and verify hypotheses concerning its functioning. We have chosen here to examine the handling of mistakes as presented in the communicative approach. In an initial phase, we propose to study the representations made by teachers of French as a foreign language and those made by their students. We will interrogate students concerning their conceptions and their attitudes about 
errors committed or encountered, and about the norms that these people employ as reference points.

In a second section, we shall see the levels at which a "linguistic consciousness" possessed by teachers and students shows itself when they are exposed to a series of utterances commonly considered mistakes, or "typical deviations", which they must then identify, classify, and evaluate. At the same time, we will ask questions about the importance of the distinction language/communication for our sample participants.

INDEX

Mots-clés : FLE, erreurs, représentations, métalangage, conscience linguistique

Keywords : FSL, French as a foreign language, mistakes, metalanguage, linguistic awareness

\section{AUTEURS}

\section{JEAN-MARC DEFAYS}

Service de didactique du FLE, Université de Liège

DEBORAH MEUNIER

Service de didactique du FLE, Université de Liège 